\title{
Entwicklung eines Lateral Flow Assays zum Nachweis von Amitriptylin in Oberflächengewässern
}

\author{
F. Schenk, J. Hutterer, K. Tondwa, C. Schanbacher, G. Gauglitz \\ Institut für Physikalische und Theoretische Chemie, Eberhard Karls Universität, Tübingen, Deutschland \\ Kontakt: guenter.gauglitz@ipc.uni-tuebingen.de
}

\section{Einleitung}

Der Konsum an Medikamenten und anderen bioaktiven Stoffen ist in Deutschland alltäglich. Allein im Jahr 2018 wurden von den gesetzlichen Krankenkassen in Deutschland über $41 \mathrm{Mrd}$. verschriebene Tagesdosen gemeldet [1]. Diese Pharmazeutika und deren Metabolite gelangen in merklichen Mengen in das Abwasser, wobei neben den Ausscheidungen der Patienten auch die unsachgemäße Entsorgung von Arzneistoffen eine signifikante Eintragsquelle darstellt. Da in den Klärwerken diese Stoffe zumeist nicht vollständig aus dem Abwasser entfernt werden können [2], werden die Rückstände als Spurenstoffe in unsere aquatische Umwelt eingetragen. In vielen Fällen handelt es sich bei diesen chemischen Belastungen der Gewässer um kleine polare organische Stoffe. Als Modellsubstanz wurde daher Amitriptylin (AMT), ein tricyclisches Antidepressivum mit einem Molekulargewicht von 277 Da gewählt. 2018 war es mit 80 Mio. verordneten Tagesdosen eines der meistverschriebenen Antidepressiva [1]. Ein möglicher Einfluss von Medikamenten und bioaktiver Stoffe auf das Ökosystem wird in standardisierten ökotoxikologischen Studien untersucht, indem die Schädigungen von ausgewählten Organismen aus den verschiedenen Trophiestufen unter Laborbedingungen bestimmt wird. Hierbei wurde gezeigt, dass Amitriptylin bei einer Konzentration von $10 \mu \mathrm{g} / \mathrm{L}$ die Mortalität von Larven des Karpfens signifikant erhöht ( $20 \%$ in den ersten 30 Lebenstagen), sowie deren Herz, Leber und Nieren geschädigt werden [3]. AuBerdem zeigte sich, dass unter anderem das Wachstum von Zebrafischen bereits von $100 \mathrm{ng} / \mathrm{L}$ Amitriptylin gehemmt wird [4]. Diese Effekte werden dem ausgelösten oxidativen Stress zugeschrieben [5].

Da der Nachweis und die Quantifizierung der Spurenstoffe in Gewässern oft kosten- und zeitintensiv sind, werden nur sehr wenige Spurenstoffe in der aquatischen Umwelt im Zuge wissenschaftlicher Fragestellungen untersucht. Zudem ist von sehr vielen Stoffen nicht bekannt, in welchen Mengen und in welcher Form sie nach der Verstoffwechselung im
Körper in die Umwelt freigesetzt werden. Um unsere Umwelt nachhaltig zu schützen, ist es zunächst wichtig, Möglichkeiten für den Nachweis anthropogener Verunreinigungen in Oberflächengewässern $\mathrm{zu}$ entwickeln. Anforderungen für eine solche Methode sind die Kosteneffizienz, kurze Zeiten zwischen Teststart und Ergebnismitteilung an den Endnutzer wie auch eine einfache Handhabung. Etablierte Methoden zur Bestimmung vom Amitriptylin sind LC- und HPLC-MS $[2,6]$ mit denen Konzentrationen von $0,5-21 \mathrm{ng} / \mathrm{L}$ in Flusswasserproben gemessen wurden. Nachteile dieser Methoden sind hohe Kosten und langwierige Probenvorbereitung. Des Weiteren gibt es voltammetrische Methoden, bei denen eine Nachweisgrenze von $33 \mu \mathrm{g} / \mathrm{L}$ erreicht werden konnte [7]. Biosensoren sind durch den einfachen Aufbau, die hohe Automatisierbarkeit sowei die spezifische Antigen-Antikörper-Reaktion vorteilhaft. Mittels reflektometrischer Interferenzspektroskopie (RIfS) konnte eine Nachweisgrenze von 0,3 $\mu \mathrm{g} / \mathrm{L}$ Amitriptylin in Puffer erzielt werden [8]. Eine weitere Methode, die die Anforderungen erfüllen kann, ist der Lateral Flow Assay (LFA). Dieser basiert auf dem Prinzip des Immunoassays, der ebenfalls auf einer Antikörper-Antigen-Reaktion basiert. Bei dieser Methode ist das Sandwich-Format üblich [9], welches aber nicht für kleine Analyten verwendet werden kann.

In dieser Studie wurde daher erstmals ein papierbasierter Bindungshemmtest zum quantitativen Nachweis von Amitriptylin in Oberflächengewässern entwickelt. Die Auswertung von LFAs ist jedoch häufig entweder nur qualitativ (Ja/Nein) oder durch Vergleich mit einer Referenzskala semiquantitativ. Daher wurden die Testlinienintensitäten mithilfe eines Matlab-basierten Algorithmus quantitativ ausgewertet. Weiterhin konnte auf die für einen Bindungshemmtest übliche Inkubationsphase verzichtet werden. Die weitere Vereinfachung des Tests, der Nutzerfreundlichkeit und der Analysendauer wird dadurch erheblich verbessert. Der Arbeitsbereich liegt im Bereich von $0,39-27,4 \mu \mathrm{g} / \mathrm{L}$. Die Übertragbarkeit des Schnelltests auf Realproben wird 
exemplarisch gezeigt. Die Wiederfindungsraten in Flusswasser liegen bei $5-25 \%$.

\section{Methoden und Materialien}

\section{Funktionsweise und Aufbau}

Der schematische Aufbau der Teststreifen ist in Abb. 1 a gezeigt. Wird die Analytflüssigkeit auf den Probenbereich (Sample Pad) aufgetragen, breitet sich die Flüssigkeit durch Kapillarkräfte auf dem Teststreifen aus. Dort interagiert sie mit den verschiedenen Testkomponenten. Zunächst gelangt die Probe auf das Konjugat Pad, wo sich mit Goldnanopartikeln markierte Amitriptylin-Antikörper befinden. Diese lösen sich und fließen zusammen mit der Probe auf die Nitrocellulosemembran. Dort befindet sich die Testlinie mit immobilisertem NortriptylinRinderserumalbumin. Solange kein AMT in der Probe enthalten ist, kann der Antikörper, entsprechend des Prinzips des Bindungshemmtests, an dieses Amitriptylinderivat binden und die volle Linienintensität ist sichtbar (Abb. 1 b). Ist Amitriptylin vorhanden, kann dieses den Antikörper blockieren und die Linienintensität nimmt $a b$ (Abb. 1 c). Nach der Testlinie befindet sich die Kontrolllinie (anti-Maus IgG), die alle mit Gold markierten Antikörper bindet und die Validität des Tests anzeigt. Das Absorbent Pad schließlich nimmt überschüssige Probenflüssigkeit auf. Somit werden größere Probenvolumina ermöglicht und unspezifische Wechselwirkungen verringert.

\section{a}

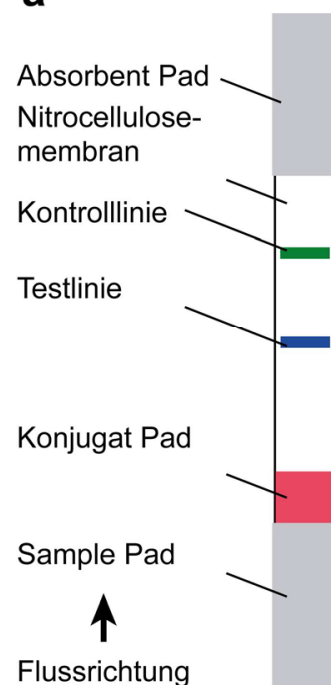

b nicht blockiert volle Testlinienintensität

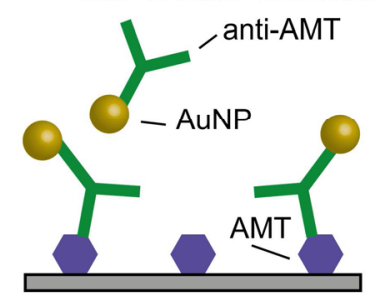

C vollständig blockiert keine Testlinienintensität

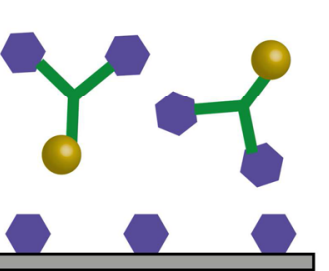

Abb. 1 a) Schematischer Aufbau der verwendeten Teststreifen. Die Flussrichtung ist von unten nach oben. b) Schematische Darstellung eines Bindungshemmtests ohne Analyt, was eine hohe Testlinienintensität auslöst und b) mit Analyt, was eine geringere Testlinienintensität zur Folge hat.

\section{Markieren der Anti-Amitriptylin-Antikörper:}

$\mathrm{Zu} 1 \mathrm{~mL}$ einer Goldkolloidlösung $(20 \mathrm{~nm}, 7$. $10^{11}$ Partikel $/ \mathrm{mL}, \mathrm{BBI}$ International, UK) werden $8 \mu \mathrm{L}$ anti-Amitriptylin $(1,2 \mu \mathrm{g} / \mathrm{mL})$ gegeben und $40 \mathrm{~min}$ inkubiert. Anschließend werden $100 \mu \mathrm{l}$ einer Rinderserumalbuminlösung $(100 \mathrm{mg} / \mathrm{mL}$ in PBS, $0,01 \mathrm{M}$, $\mathrm{pH} 7,4)$ hinzugefügt, um noch vorhandene Bindungsstellen abzusättigen. Nach einer weiteren Inkubationszeit von 20 min wird die Lösung zentrifugiert $\left(20 \mathrm{~min}, 13.000 \mathrm{~min}^{-1}\right)$. Der Überstand wird verworfen und das Nanopartikelpellet wird in einer RSA-Lösung $(0,1 \mathrm{mg} / \mathrm{mL}$ in PBS) resuspendiert. Dieser Waschschritt wird noch zwei Mal wiederholt. Nach Zugabe von $100 \mathrm{mg}$ Saccharose wird das Konjugat bis zur Verwendung bei $4{ }^{\circ} \mathrm{C}$ gelagert.

\section{Testkomponenten:}

Die Sample und Absorbent Pads bestehen aus Cellulose $(11,5 \times 5 \mathrm{~mm}$, SureWick CFSP, Merck Millipore, Darmstadt, Deutschland), wobei erstere in einer Tween ${ }^{\circledR} 20$-Lösung ( $10 \mathrm{~mL}, 2 \mu \mathrm{L} / \mathrm{mL}$ in PBS) für $1 \mathrm{~h}$ imprägniert und anschließend bei $37^{\circ} \mathrm{C}$ getrocknet werden. Die Konjugat Pads (Glasfaser, SureWick GFCP, Merck Millipore, Darmstadt, Deutschland) werden $1 \mathrm{~h}$ mit einer RSA-Lösung $(10 \mathrm{~mL}, 10$ $\mathrm{mg} / \mathrm{mL}$ in PBS) imprägniert und bei $37^{\circ} \mathrm{C}$ getrocknet. Anschließend werden diese mit $15 \mu \mathrm{L}$ der antiAmitriptylin-Goldnanopartikel (Konjugat) versehen und bei $37^{\circ} \mathrm{C}$ getrocknet. Die Testmembran (CN 140, UniSart $\circledast$, Fließgeschwindigkeit: 110-165 s/4 $\mathrm{cm}$, Sartorius AG, Göttingen, Deutschland) wurde durch einen BioOdyssey ${ }^{\mathrm{TM}}$ Calligrapher ${ }^{\mathrm{TM}}$ Miniarrayer (Bio-Rad, Hercules USA) mit der Testlinie (1 mg/mL, Nortriptylin-RSA, CalBioreagents, San Mateo, USA) und der Kontrolllinie $(1 \mathrm{mg} / \mathrm{mL}$, Ziege anti-Maus IgG, polyklonal, Antikörper-Online, Aachen, Deutschland) versehen, für $1 \mathrm{~h}$ bei $37^{\circ} \mathrm{C}$ immobilisiert und in $5 \mathrm{~mm}$ breite Streifen geschnitten. Durch die auf der Membran befindlichen Klebestreifen werden die Komponenten, wie in Abb. 1 a zu sehen, zusammengesetzt.

\section{Testverlauf:}

Die Teststreifen werden mit $150 \mu \mathrm{L}$ der mit AMT versetzten Probelösungen versetzt (AmitriptylinHydrochlorid, LKT Labs, St. Paul, USA) und nach 30 min fotografiert (Olympus E-1 Kamera, Blende: f/11, ISO-Wert: 100). Flusswasserproben wurden aus der Steinlach, Tübingen entnommen.

\section{Auswertung:}

Zunächst wird mit dem Programm ImageJ ein Weißabgleich durchgeführt und die Bilder kontrastver- 
stärkt, um Einflüsse der Beleuchtung und des Hintergrundes zu minimieren. Die Teststreifenintensitäten werden mittels eines Matlab-Programms ausgewertet. Der Algorithmus ist in Abb. 2 schematisch gezeigt. Zur Bestimmung der region of interest (ROI) werden im HSV-Farbraum nur Pixel ausgewählt, die sich in einem definierten Farb- und Intensitätsbereich befinden. Die ausgewählten Pixel werden im RGB-Farbraum invertiert, damit eine starke Färbung mit einer hohen Intensität korreliert. Der Mittelwert der Werte des grünen Kanals werden als Testlinienintensität berechnet.

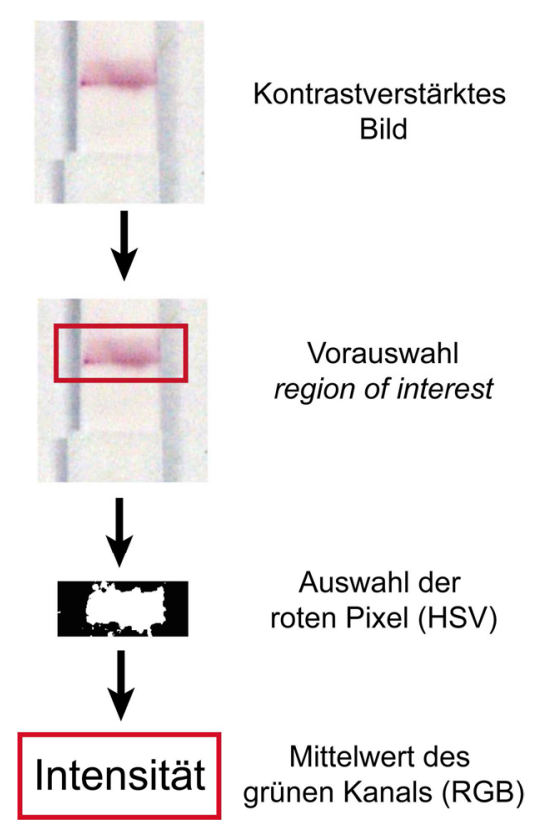

Abb. 2 Workflow des Auswertungsalgorithmus

\section{Ergebnisse und Diskussion}

Im Rahmen dieser Studie wurde die Methode des Lateral Flow Assays zum Nachweis von Amitriptylin erprobt. Dabei wurde für den Immunoassay auf das bereits für RIfS etablierte Format des Bindungshemmtests zurückgegriffen [8]. In vorherigen Arbeiten wurden mehrere Nitrocellulosemembranen und Größen der Goldnanopartikel verglichen, wobei die Wahl auf die CN140-Membran von Sartorius bzw. die $20 \mathrm{~nm}$ Partikel fiel. Außerdem wurde im Rahmen von Vorarbeiten ein Flokkulationstest durchgeführt, um die optimale Menge an Antikörpern zur Herstellung des Konjugats festzustellen.

In Abb. 3 ist die Kalibrierung von Amitriptylin in PBSPuffer gezeigt. Eine logistische Funktion wurde an die Messwerte angepasst, deren Parameter in Tab. 1 dargestellt sind. Der Arbeitsbereich reicht von 0,39 bis $27,4 \mu \mathrm{g} / \mathrm{L}$ und ist definiert als die Konzentrationen die 90 bzw. $10 \%$ der Testintensität darstel- len. Es zeigt sich, dass die Intensität in Abhängigkeit der Konzentration sehr gut mit der für einen Immunoassay typischen logistischen Funktion übereinstimmt. Hier zeigt sich, dass die vorliegende Methode bereits mit voltammetrischen Verfahren [7] und etablierten Biosensoren [8] vergleichbar ist. Bei Letzterem wurde außerdem eine gesonderte Inkubationsphase für den Bindungshemmtest verwendet, auf die in der vorliegenden Studie verzichtet wurde. Trotzdem muss der Arbeitsbereich weiter verbessert werden, damit auch geringe AMT-Konzentrationen im Wasser im Bereich von ng/L erfasst werden können.

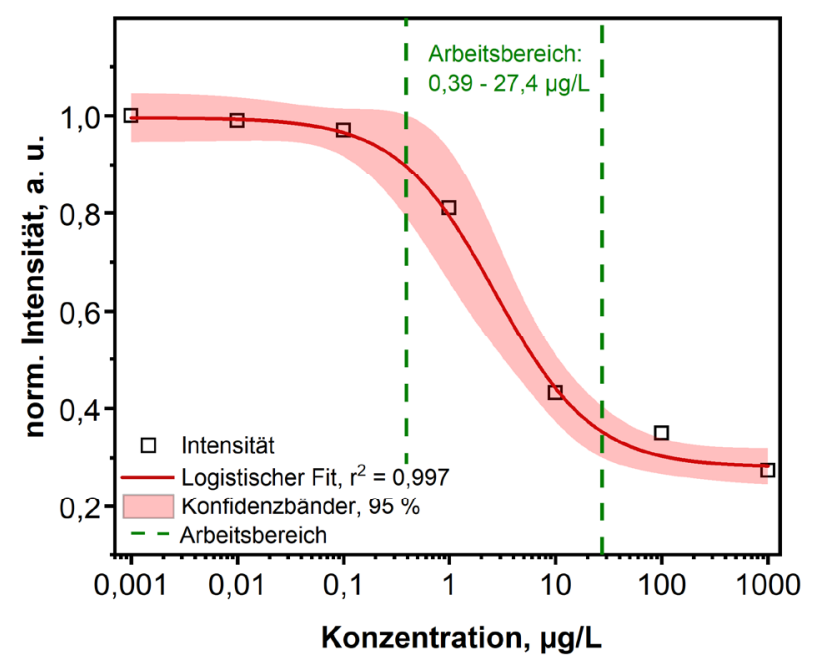

Abb. 3 Normierte Testlinienintensitäten als Funktion der AMTKonzentration. Zur Kalibration wurde eine logistische Funktion zur Anpassung (siehe Tab. 1) verwendet. Die Konfidenzbänder (95\%, hellrot) sowie der Arbeitsbereich (grün) sind eingezeichnet.

Für den Einsatz in Realproben ist nicht nur der Arbeitsbereich, sondern auch die Selektivität bzw. die Spezifität wichtig. Verglichen zu AntiproteinAntikörper weisen Antikörper gegen kleine Analyten eine hohe Kreuzreaktivität gegenüber strukturell verwandten Stoffen auf [10]. Daher wurde die Kreuzreaktivität gegen die tricyclischen Antidepressiva Clomipramin, Doxepin, Imipramin, Opipramol sowie das Protein Ovalbumin untersucht.

Tab. 1 Für den logistischen Fit verwendete Formel, die Werte der Parameter und die dazugehörigen Standardfehler

\begin{tabular}{|c|c|c|}
\hline \multirow{2}{*}{ Formel } & \multicolumn{2}{|c|}{$y=\frac{A_{1}-A_{2}}{1+\left(\frac{x}{x_{0}}\right)^{p}}+A_{2}$} \\
\hline Parameter & Wert & Standardfehler \\
\hline$A_{1}$ & 0,997 & 0,018 \\
\hline$A_{2}$ & 0,281 & 0,014 \\
\hline$x_{0}$ & 2,68 & 0,74 \\
\hline$p$ & 0,94 & 0,18 \\
\hline
\end{tabular}


Die Ergebnisse sind in Abb. 4 dargestellt. Es zeigt sich, dass Clomipramin und Opipramol den Antikörper in einem vergleichbaren Maß blockieren wie Amitriptylin. Doxepin und Imipramin hingegen haben eine geringere Fähigkeit den Antikörper zu blockieren. Aufgrund dieser Kreuzreaktivitäten könnte sich der LFA daher auch für die Bestimmung eines Summenparameters an tricyclischen Antidepressiva eignen, die alle strukturell verwandt sind und sich ähnlich auf die Umwelt auswirken.

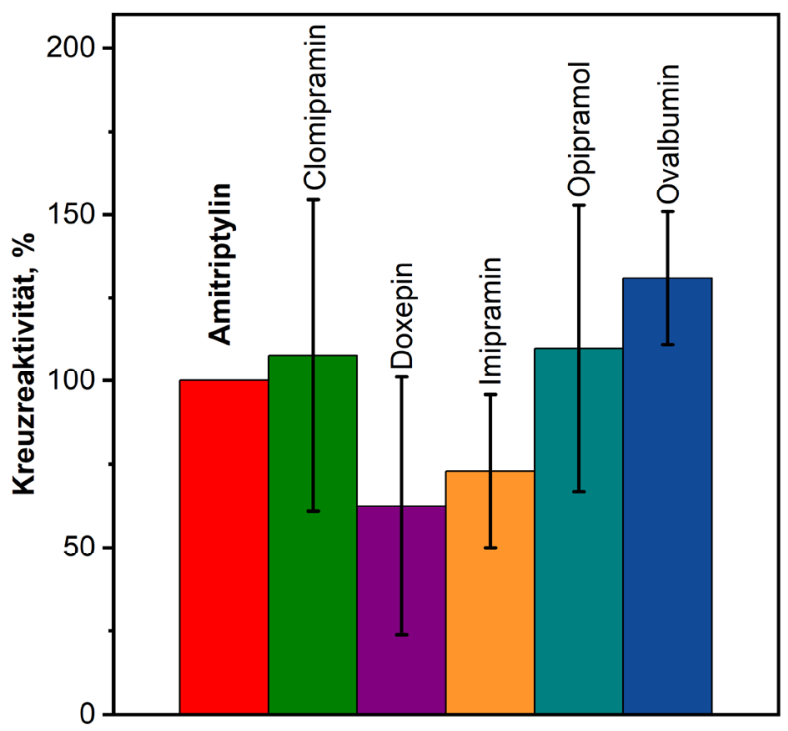

Abb. 4 Kreuzreaktivitäten des Lateral Flow Assays gegen die tricyclischen Antidepressiva Clomipramin (grün), Doxepin (violett), Imipramin (orange) und Opipramol (türkis). Außerdem das Protein Ovalbumin (blau)

Zuletzt wurde die prinzipielle Übertragbarkeit auf Realproben in Form von Flusswasser erprobt. Hierfür wurde Wasser vor und nach einer Kläranlage entnommen und mit $7 \mu \mathrm{g} / \mathrm{L}$ AMT aufgestockt. Die Ergebnisse sind in Abb. 5 dargestellt. Vor dem Klärwerk zeigt sich eine Wiederfindungsrate von $25 \%$, während für den Ablauf $5 \%$ bestimmt wurden. Der Unterschied kann aufgrund der durch die Kläranlage eingeführten Matrixeffekte oder der Streuung bei der Produktion der Teststreifen aufgetreten sein. Für die Verwendung eines Immunoassays in realen Einsatzbedingungen sind Wiederfindungsraten von 80 $120 \%$ akzeptabel. Diese Abweichung könnte durch Verbesserung der Chargenreproduzierbarkeit der Teststreifen oder durch weitere Optimierung der Testkomponenten minimiert werden, um verlässliche Gewässermessungen durchzuführen.

\section{Fazit und Ausblick}

Es wurde gezeigt, dass mittels eines LFAs die Messung von kleinen, polaren Molekülen möglich ist. Dies wurde am Beispiel von Amitriptylin mittels eines
Bindungshemmtests gezeigt. Dabei konnte auf eine Inkubationsphase verzichtet werden und somit Testdauer und -aufwand erheblich verbessert werden. Zur Quantifizierung wurden die fotografierten Teststreifen mittels eines Matlab-basierten Algorithmus ausgewertet. Ein mit anderen Sensoren vergleichbarer Arbeitsbereich konnte erzielt werden. Wie für kleine Analyten bekannt, zeigt der Antikörper Kreuzreaktivitäten gegen strukturverwandte Stoffe. Die Messung von aufgestockten Flusswasserproben ist möglich, allerdings müssen die Wiederfindungsraten weiter verbessert werden. Vor allem Parameter, wie die Menge an Konjugat oder die Menge an immobilisierten Antigenen haben einen großen Einfluss auf Testintensität, Nachweisgrenzen und die Reproduzierbarkeit. Daher muss das Verfahren durch Optimierungen dieser Parameter noch weiter verbessert werden.

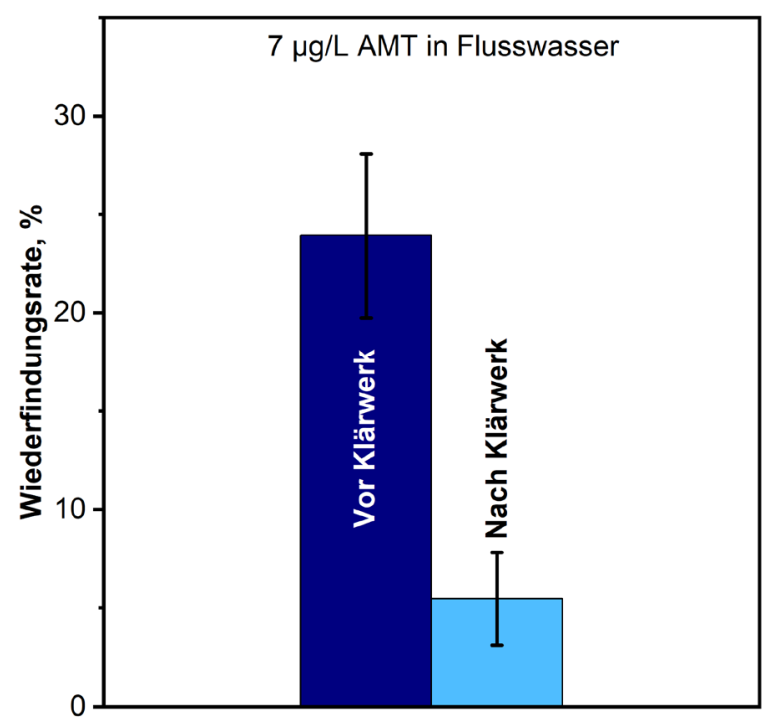

Abb. 5 Wiederfindungsraten in aufgestocktem Flusswasser $(7$ $\mu \mathrm{g} / \mathrm{L}$ AMT) in Flusswasser oberhalb und unterhalb des Klärwerks, bestimmt nach [11]

\section{Literatur}

1. Schwabe U, Paffrath D, Ludwig W-D, Klauber J (2019) Arzneiverordnungs-Report 2019. Springer Berlin Heidelberg, Berlin, Heidelberg

2. Lajeunesse A, Gagnon C, Sauvé S (2008) Determination of basic antidepressants and their $\mathrm{N}$-desmethyl metabolites in raw sewage and wastewater using solid-phase extraction and liquid chromatography-tandem mass spectrometry. Anal Chem 80:5325-5333

3. Sehonova P, Plhalova L, Blahova J, Doubkova V, Marsalek $P$, Prokes $M$, Tichy $F$, Skladana $M$, Fiorino $E$, Mikula $P$, Vecerek $V$, Faggio $C$, Svobodova Z (2017) Effects of selected tricyclic antidepressants on early-life stages of common 
carp (Cyprinus carpio). Chemosphere 185:10721080

4. Yang M, Qiu W, Chen J, Zhan J, Pan C, Lei X, Wu $M$ (2014) Growth inhibition and coordinated physiological regulation of zebrafish (Danio rerio) embryos upon sublethal exposure to antidepressant amitriptyline. Aquat Toxicol 151:68-76

5. Moreno-Fernández $\mathrm{AM}$, Cordero MD, de Miguel $M$, Delgado-Rufino $M D$, Sánchez-Alcázar JA Navas P (2008) Cytotoxic effects of amitriptyline in human fibroblasts. Toxicology 243:51-58

6. Kasprzyk-Hordern B, Dinsdale RM, Guwy AJ (2008) The occurrence of pharmaceuticals, personal care products, endocrine disruptors and illicit drugs in surface water in South Wales, UK. Water Res 42:3498-3518

7. Marco JP, Borges KB, Tarley CRT, Ribeiro ES, Pereira AC (2013) Development and application of an electrochemical biosensor based on carbon paste and silica modified with niobium oxide, alumina and $\mathrm{DNA}\left(\mathrm{SiO}_{2} / \mathrm{Al}_{2} \mathrm{O}_{3} / \mathrm{Nb}_{2} \mathrm{O}_{5} / \mathrm{DNA}\right)$ for amitriptyline determination. $\mathrm{J}$ Electroanal Chem 704:159-168

8. Krieg AK, Gauglitz G (2015) Ultrasensitive LabelFree Immunoassay for Optical Determination of Amitriptyline and Related Tricyclic Antidepressants in Human Serum. Anal Chem 87:8845-8850

9. Schenk F, Weber P, Vogler J, Hecht L, Dietzel A, Gauglitz G (2018) Development of a paper-based lateral flow immunoassay for simultaneous detection of lipopolysaccharides of Salmonella serovars. Anal Bioanal Chem 410:863-868

10. Holtzapple CK, Buckley SA, Stanker LH (1997)

Production and Characterization of Monoclonal Antibodies against Sarafloxacin and CrossReactivity Studies of Related Fluoroquinolones. J Agric Food Chem 45:1984-1990

11. Burns DT, Danzer K, Townshend A (2002) IUPAC Recommendations: Use of the Terms "Recovery" and "Apparent Recovery" in Analytical Procedures. Pure Appl Chem 74:2201-2205 